\title{
Coplanar transitions based on aluminum nitride interposer substrate for terabit transceivers
}

Dong, Yunfeng; Johansen, Tom Keinicke; Zhurbenko, Vitaliy; Hanberg, Peter Jesper

Published in:

2017 47th European Microwave Conference (EuMC)

Link to article, DOI:

10.23919/EuMC.2017.8230924

Publication date:

2017

Document Version

Peer reviewed version

Link back to DTU Orbit

Citation (APA):

Dong, Y., Johansen, T. K., Zhurbenko, V., \& Hanberg, P. J. (2017). Coplanar transitions based on aluminum nitride interposer substrate for terabit transceivers. In 2017 47th European Microwave Conference (EuMC) (pp. 620-623). IEEE. https://doi.org/10.23919/EuMC.2017.8230924

\section{General rights}

Copyright and moral rights for the publications made accessible in the public portal are retained by the authors and/or other copyright owners and it is a condition of accessing publications that users recognise and abide by the legal requirements associated with these rights.

- Users may download and print one copy of any publication from the public portal for the purpose of private study or research.

- You may not further distribute the material or use it for any profit-making activity or commercial gain

- You may freely distribute the URL identifying the publication in the public portal 


\section{Coplanar Transitions Based on Aluminum Nitride Interposer Substrate for Terabit Transceivers}

\author{
Yunfeng Dong, Tom K. Johansen, Vitaliy Zhurbenko \\ Electromagnetic System, Department of Electrical Engineering \\ Technical University of Denmark \\ DK-2800 Kgs. Lyngby, Denmark \\ yundon/tkj/vz@elektro.dtu.dk
}

\author{
Peter Jesper Hanberg \\ DTU Danchip \\ Technical University of Denmark \\ DK-2800 Kgs. Lyngby, Denmark \\ jehan@danchip.dtu.dk
}

\begin{abstract}
This paper presents two types of coplanar transitions based on aluminum nitride (AIN) substrate for interposer designs of terabit transceivers. The designs of coupled coplanar waveguide (CCPW), coupled line, coplanar waveguide (CPW), and coplanar stripline (CPS) based on AIN substrate are explained. The effects of absorber layer and wire bonding bridges are described. Two types of coplanar transitions are designed and simulated in back-to-back configuration with wire bonding bridges. When driven by differential signal pair, the proposed CCPW-to-coupled line transition in back-to-back configuration with wire bonding bridges achieves a simulated return loss of 11 $\mathrm{dB}$ and insertion loss of $2 \mathrm{~dB}$ up to $110 \mathrm{GHz}$. As for single-ended signals, a CPW-to-CPS transition in back-to-back configuration with wire bonding bridges has been designed, fabricated, and measured. The fabricated CPW-to-CPS transition can provide a $-3 \mathrm{~dB}$ transmission bandwidth up to $80 \mathrm{GHz}$ with associated return loss better than $12 \mathrm{~dB}$.
\end{abstract}

\section{INTRODUCTION}

With the ever increasing demand worldwide for highspeed communication and comprehensive multimedia services, the existing data transmission systems especially the currently used edge switches and datacenter gateways are being challenged and approaching their design limits. As an alternative, the optical Ethernet has been considered to be an ideal candidate for the next-generation high-speed data transmission system [1]. Besides, the current implementation of $100 \mathrm{Gbit} / \mathrm{s}$ modulation scheme based on dual-polarization quadrature phase-shift keying (DP-QPSK) is saturated. The next migration from $100 \mathrm{Gbit} / \mathrm{s}$ to $400 \mathrm{Gbit} / \mathrm{s}$ and further on to $1 \mathrm{Tbit} / \mathrm{s}$ optical line card products based on higher-order quadrature amplitude modulation (QAM) should take place in the near future [2].

PANTHER is an European project and its name comes from the abbreviation of PAssive and electrooptic (EO) polymer photonics and indium phosphide (InP) electronics iNtegration for multi-flow Terabit transceivers at edge softwaredefined networking (SDN) switcHes and datacentER gateways. The goal of the project is to develop transceivers that have terabit throughput and at the same time the throughput can be easily tuned to fit the new IP traffic due to its dynamic nature. By using dual-polarization (DP) 64-QAM and 64 Gbaud operation rate, the overall capacity of the PANTHER transceivers can go up to $1.536 \mathrm{Tbit} / \mathrm{s}$ [2].

Fig. 1 shows the envisaged PANTHER transmitter based on 3D hybrid integration scheme of photonic and electronic components. The drivers (DRVs) in the integration are based

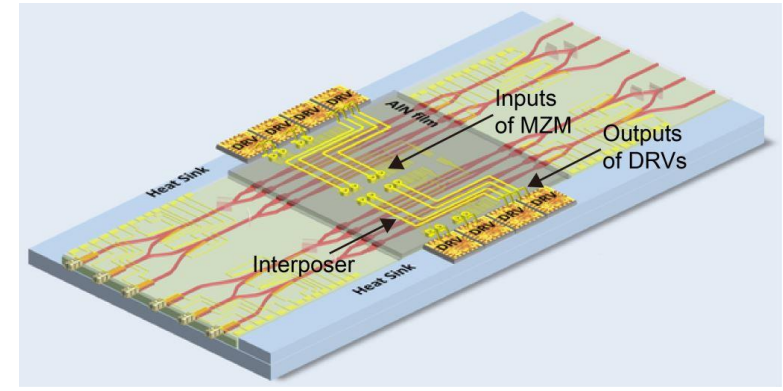

Fig. 1. The envisaged transmitter based on 3D hybrid integration of photonic and electronic components (from [3])

on InP double heterojunction bipolar transistor (InP-DHBT) technology which work as digital-to-analog converters. Both differential and single-ended signals are supported by the DRVs. The signals go out of the DRVs and then coupled into the interposer based on AlN substrate. The interposer is connected to an InP Mach-Zehnder modulator (MZM) through via holes. The heat generated by each DRV is approximately $3 \mathrm{~W}$. A copper tungsten $(\mathrm{CuW})$ layer is used as the heat sink under the DRVs and MZM. The bandwidth of the interposer should be at least $64 \mathrm{GHz}$ in order to support a 64 Gbaud operation rate of the transceiver. A methodology of 3D hybrid integration scheme for PANTHER terabit transceiver was reported in [4].

In this work, contributed to PANTHER, CCPW-to-coupled line and CPW-to-CPS transitions are designed and analyzed by using High Frequency Structural Simulator (HFSS). The transitions are needed for the interposer design due to different signal driving schemes between the outputs of DRVs and the inputs of the InP MZM. In Section II, the designs of CCPW, coupled line, and CCPW-to-coupled line transition based on AlN substrate are explained. Besides, the effects of absorber layer and wire bonding bridges are described. In Section III, the designs of CPW, CPS, and CPW-to-CPS transition based on AlN substrate are demonstrated. In Section IV, the fabrication and measurement results of the proposed CPW-to-CPS transition based on AlN substrate are shown and compared with simulation results.

\section{Coupled Coplanar WaVeguide-To-Coupled Line TRANSITION}

When the signals are transmitted differentially, the outputs of the DRVs shown in Fig. 1 are in a ground-signal- 


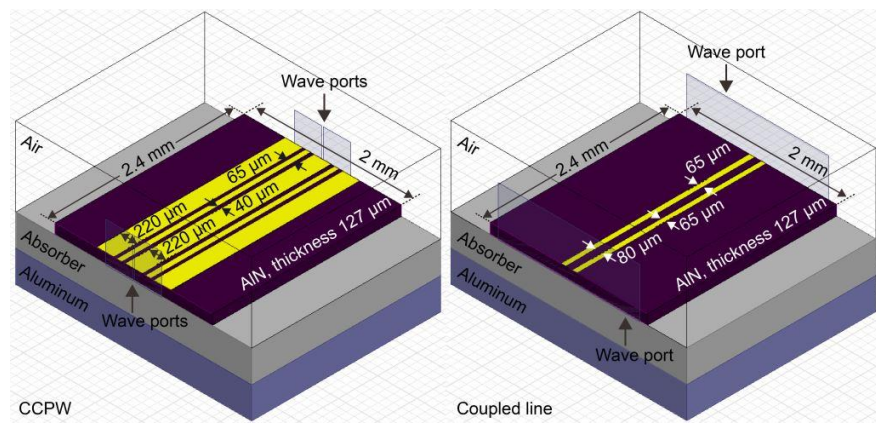

Fig. 2. CCPW and coupled line based on AlN substrate and absorber.

ground-signal-ground (GSGSG) configuration where the two complementary signals are transmitted $180^{\circ}$ out of phase. The information is transmitted by the difference between the two signals and the interference during the transmission is cancelled. In order to match the GSGSG structure, the socalled coupled coplanar waveguide (CCPW) is implemented. The inputs of the InP MZM at the end of the interposer work in a signal-signal (SS) configuration which requires coupled line connections. Under this circumstance, the CCPW-to-coupled line transition on the interposer is essential for driving the differential signal pair.

As it shown in Fig. 2, the simulation structure of the designed CCPW is based on AlN substrate and absorber. The width of the signal traces is $65 \mu \mathrm{m}$ and the width of the ground traces is $220 \mu \mathrm{m}$ with a gap width of $40 \mu \mathrm{m}$. The material of the conductor is gold and the thickness is 1.2 $\mu \mathrm{m}$. The AlN substrate is $2.4 \mathrm{~mm}$ in length and $2 \mathrm{~mm}$ in width with a thickness of $127 \mu \mathrm{m}$. An absorber layer is added between the AIN substrate and the bottom heat sink layer in order to suppress parasitic modes. Wave ports are used as the excitation scheme and the edges of the wave ports touch the ground traces. The differential excitations are assigned to the two signal traces in the simulation. As for the designed coupled line structure in Fig. 2, the length of the signal traces is 2.4 $\mathrm{mm}$ and the width is $65 \mu \mathrm{m}$ with a $80 \mu \mathrm{m}$ gap between the two signal traces. The substrate and the gold conductor layer are the same as that used for the CCPW. Wave ports are used again and they have to be wide enough to generate the correct port field. There is no ground trace for coupled line and the two signal traces are assigned to be a differential pair in the simulation.

The simulation results of the designed CCPW and coupled line based on AlN substrate are shown in Fig. 3. PolyOxyMethylene (POM) is used as the material of the absorber. The thickness of the absorber layer is $3.5 \mathrm{~mm}$. The dielectric constant $\left(\epsilon_{r}\right)$ and dissipation factor $(\tan \delta)$ of POM are set to be 3.8 and 0.06 , respectively, in the simulation. The dash lines in Fig. 3 show the simulation results of the designed CCPW and coupled line without absorber while the solid lines are the simulation results when the POM absorber layer is added. With POM absorber layer the designed CCPW and coupled line show smooth transmissions up to $110 \mathrm{GHz}$ with $1 \mathrm{~dB}$ insertion loss and $15 \mathrm{~dB}$ return loss. Although the notches appear when there is no absorber layer, they are not significant. This is due to the intrinsic property of a differential signal pair.

Based on the CCPW and coupled line structures, the proposed CCPW-to-coupled line transition in back-to-back
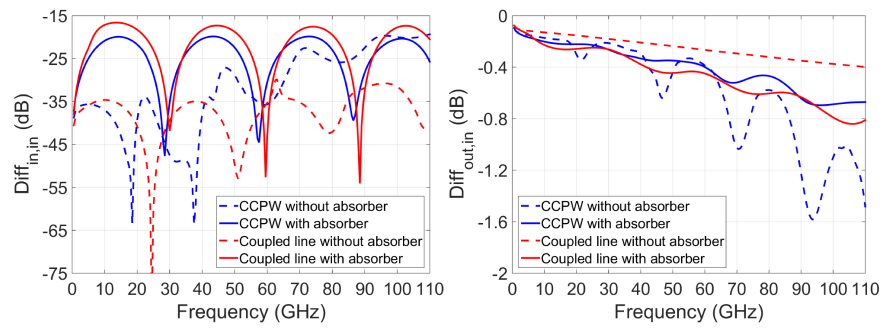

Fig. 3. Simulation results of CCPW and coupled line based on AIN substrate with (solid lines) and without absorber layer (dash lines).

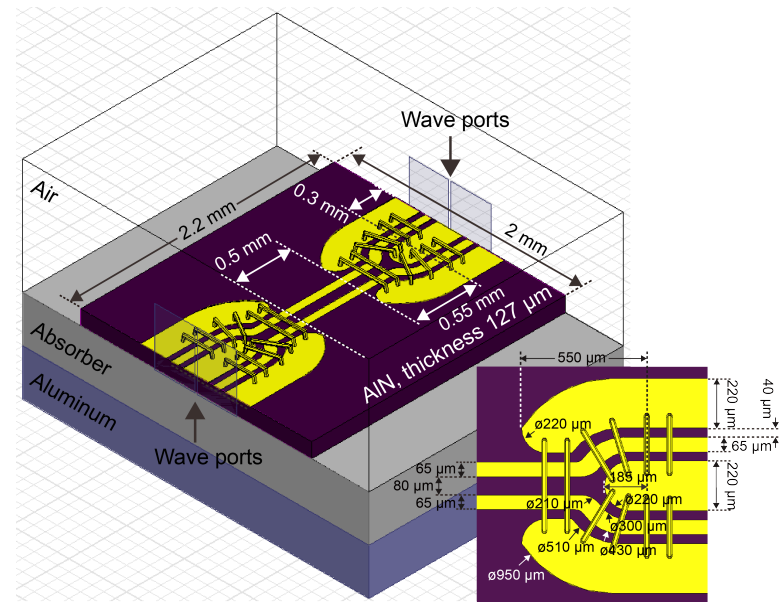

Fig. 4. CCPW-to-Coupled line transition in back-to-back configuration.

configuration is shown in Fig. 4. The AlN substrate is 2.2 $\mathrm{mm}$ in length and $2 \mathrm{~mm}$ in width with a thickness of 127 $\mu \mathrm{m}$. The thickness of the POM absorber layer is $3.5 \mathrm{~mm}$. The lengths of the CCPW and coupled line are $0.3 \mathrm{~mm}$ and 0.5 $\mathrm{mm}$, respectively. The transition is $550 \mu \mathrm{m}$ in length where the center ground trace of the CCPW terminates smoothly and the two signal traces move towards each other forming a ground-signal-signal-ground (GSSG) structure. At the end of the GSSG structure, the two ground traces on the sides are faded out and become a SS structure which is also the starting point of the coupled line. Wire bonding bridges are used all the way along the transition to suppress parasitic modes. The diameter of the wire bonding bridges is $25 \mu \mathrm{m}$ and the height is $50 \mu \mathrm{m}$.

The red solid lines in Fig. 5 show the simulation results of the proposed CCPW-to-coupled line transition with wire bonding bridges when driven by a differential signal pair. The return loss is better than $11 \mathrm{~dB}$ and the insertion loss is less than $2 \mathrm{~dB}$ up to $110 \mathrm{GHz}$. The blue solid lines in Fig. 5 show the simulation results when there is no wire bonding bridge. It is used as the reference which shows the improvement by using wire bonding bridges at the transition structures. The proposed CCPW-to-coupled line transition should be sufficient to support a bandwidth of $64 \mathrm{GHz}$ since there is only one CCPW-to-coupled line transition for the interposer design.

\section{COPlanar WAVEguide-To-Coplanar Stripline TRANSITION}

When the signals are transmitted single-ended, the outputs of the DRVs shown in Fig. 1 are in a ground-signal-ground 

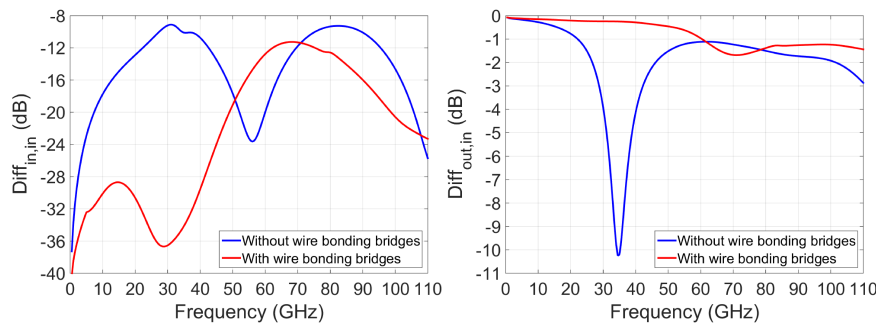

Fig. 5. Simulation results of CCPW-to-Coupled line transition in back-toback configuration with (red solid lines) and without wire bonding bridges (bule solid lines).
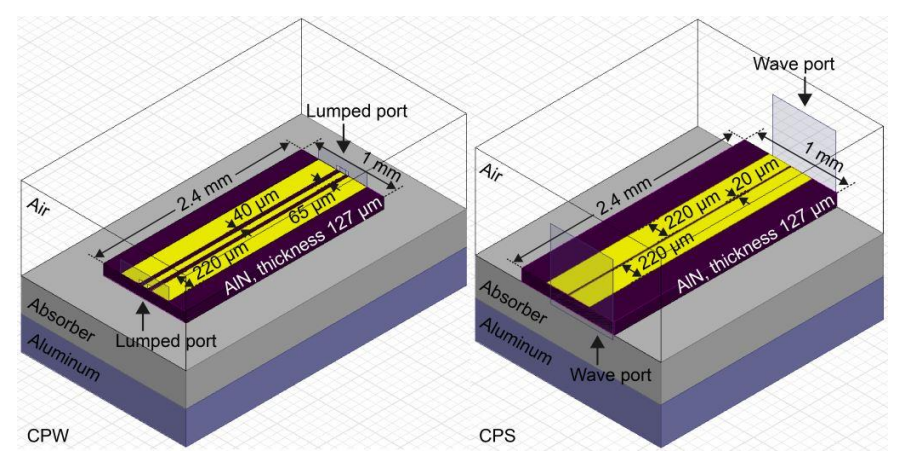

Fig. 6. CPW and CPS based on AlN substrate and absorber.

(GSG) configuration which is suitable for CPW. The inputs of the InP MZM at the end of the interposer work in a groundsignal (GS) configuration which does not match the GSG configuration of the DRVs. Under this circumstance, the CPWto-CPS transition is essential for the interposer connecting the output of the DRVs to the input of the InP MZM. A CPW-toCPS transition was reported in [5] up to $55 \mathrm{GHz}$.

As it shown in Fig. 6, the simulation structure of the designed CPW is based on AlN substrate and absorber. The substrate, absorber, and the gold conductor layer are the same as that used for the CCPW. The CPW is designed to have a characteristic impedance of $50 \Omega$. The width of the signal trace is $65 \mu \mathrm{m}$ and the width of the ground trace is $220 \mu \mathrm{m}$ with a gap width of $40 \mu \mathrm{m}$. The AlN substrate is $2.4 \mathrm{~mm}$ in length and $1 \mathrm{~mm}$ in width with a thickness of $127 \mu \mathrm{m}$. Lumped ports with vertical perfect electric conductor (PEC) bridges are used as the excitation scheme in the simulation. The PEC bridge touch the ground traces and the port is assigned to a sheet between the signal trace and the PEC bridge. The inductance of the port sheet is calibrated out from the simulation results. As for the designed CPS in Fig. 6, both signal trace and ground trace are $220 \mu \mathrm{m}$ in width with a gap of $20 \mu \mathrm{m}$. Wave ports are used as the excitation scheme where the edges of the wave ports touch the ground trace which generate excitations in a GS configuration.

Fig. 7 shows the simulation results of the designed CPW and CPS based on AlN substrate. The blue lines in Fig. 7 show the simulation results of the designed CPW with and without POM absorber layer. In both situations, the designed CPW shows smooth transmissions up to $110 \mathrm{GHz}$ with $1 \mathrm{~dB}$ insertion loss and $17 \mathrm{~dB}$ return loss. When the signal is transmitted by $\mathrm{CPW}$ mode, the energy is mainly coupled in the gaps between the signal trace and the ground traces. Besides, the length of the designed CPW is $2.4 \mathrm{~mm}$ which is relatively small compared
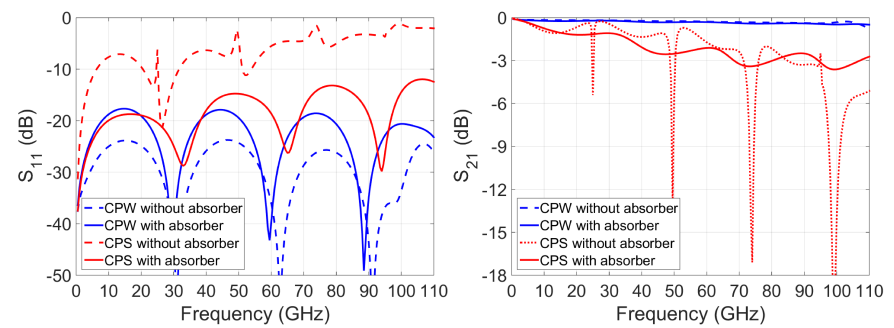

Fig. 7. Simulation results of CPW and CPS based on AlN substrate with (solid lines) and without absorber layer (dash lines).

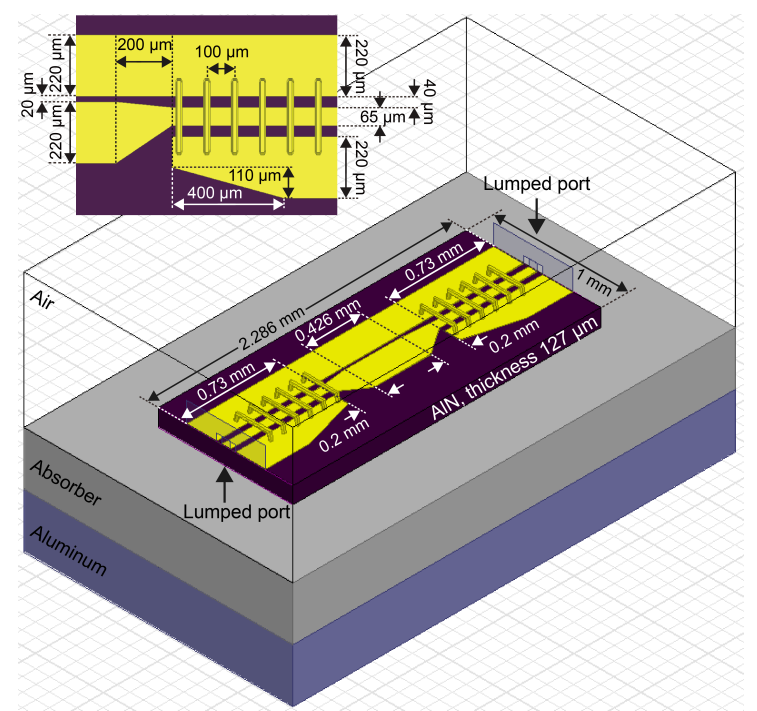

Fig. 8. CPW-to-CPS transition in back-to-back configuration.

with the wavelength at $110 \mathrm{GHz}$. As a result, the designed CPW shows a smooth transmission without the absorber layer. If the length of the designed CPW increases to $5 \mathrm{~mm}$, notches will appear starting at $43 \mathrm{GHz}$ and in that case either absorber layer or ground vias need to be added in order to suppress parasitic modes. As the red lines shown in Fig. 7, the designed CPS starts to resonate and the notches appear from $25 \mathrm{GHz}$ when there is no absorber layer. With the absorber layer, a 3 $\mathrm{dB}$ bandwidth of $68 \mathrm{GHz}$ is achieved for the designed CPS. The return loss is better than $12 \mathrm{~dB}$ up to $110 \mathrm{GHz}$.

Based on the CPW and CPS structures, the proposed CPWto-CPS transition in back-to-back configuration is shown in Fig. 8. The AlN substrate is $2.286 \mathrm{~mm}$ in length and $1 \mathrm{~mm}$ in width with a thickness of $127 \mu \mathrm{m}$. The thickness of the POM absorber layer is $3.5 \mathrm{~mm}$. The back-to-back structure consists of two CPWs with a length of $0.73 \mathrm{~mm}$, a CPS in the middle with a length of $0.426 \mathrm{~mm}$, and two transitions with a length of $0.2 \mathrm{~mm}$. Wire bonding bridges are added close to the transition between two ground traces in order to suppress parasitic modes. The diameter of the wire bonding bridges is $25 \mu \mathrm{m}$. The height of the wire bonding bridges is $50 \mu \mathrm{m}$ and the length is $255 \mu \mathrm{m}$. The distance between two wire bonding bridges is $100 \mu \mathrm{m}$. One of the ground traces is tapered in and the signal trace is tapered out guiding electromagnetic wave from CPW mode to CPS mode smoothly. The lengths of the tapered structure on CPW and CPS are $400 \mu \mathrm{m}$ and $200 \mu \mathrm{m}$, respectively.

The red solid lines in Fig. 9 show the simulation results 

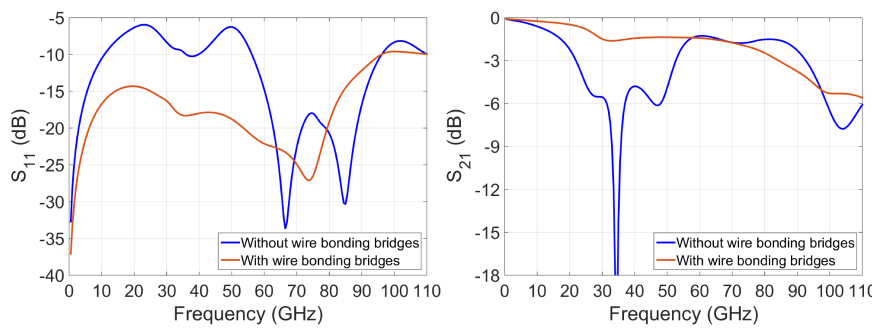

Fig. 9. Simulation results of CPW-to-CPS transition in back-to-back configuration with (red solid lines) and without wire bonding bridges (bule solid lines).

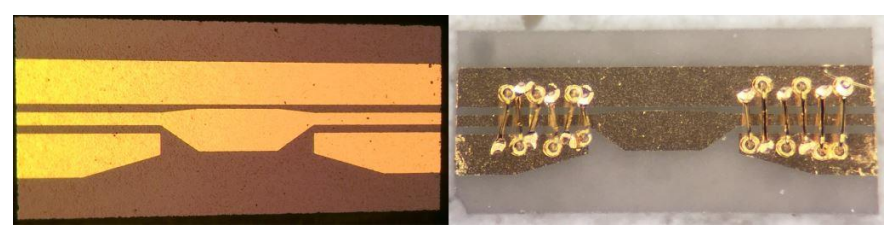

Fig. 10. Fabricated CPW-to-CPS transition in back-to-back configuration based on AlN substrate.

of the proposed CPW-to-CPS transition with wire bonding bridges. The return loss is better than $14 \mathrm{~dB}$ and the insertion loss is less than $3 \mathrm{~dB}$ up to $84 \mathrm{GHz}$. The blue solid lines in Fig. 9 show the simulation results when there is no wire bonding bridge. By adding wire bonding bridges the $3 \mathrm{~dB}$ bandwidth of the proposed CPW-to-CPS transition increases from $22 \mathrm{GHz}$ to $84 \mathrm{GHz}$. The simulation structure in Fig. 8 contains two CPW-to-CPS transitions and there is only one transition for the interposer design which should be sufficient to support a bandwidth of $64 \mathrm{GHz}$.

\section{FABRICATION AND EXPERIMENTAL RESULTS}

In order to prove the designs, the proposed CPW-to-CPS transition shown in Fig. 8 was fabricated. The fabricated transition is based on AIN substrate with a thickness of $127 \mu \mathrm{m}$. According to the supplier, the $\epsilon_{r}$ and $\tan \delta$ of the material are 8.6 and 0.001 , respectively. The skin depth for gold conductor at $110 \mathrm{GHz}$ is around $0.23 \mu \mathrm{m}$ and the thickness of the fabricated gold conductor layer is $1.2 \mu \mathrm{m}$ which also guarantees reasonable performances at low frequencies. The fabricated CPW-to-CPS transition with and without wire bonding bridges are shown in Fig. 10. The wire bonding bridges were made at Danchip (National Center for Micro- and Nanofabrication in Denmark). The diameter of the wire bonding is $25 \mu \mathrm{m}$ and the material is gold.

The fabricated CPW-to-CPS transition was characterized on-wafer using a probe station, a network analyzer and two GSG probes with $125 \mu \mathrm{m}$ pitch. The measurement results are shown in Fig. 11 and compared with the simulation results. The blue solid lines show the measurement results of the fabricated CPW-to-CPS transition with POM absorber. Compared with the blue dash lines, a reasonable agreement between simulation and measurement has been achieved. The measured return loss is better than $12 \mathrm{~dB}$ and the insertion loss is less than $3 \mathrm{~dB}$ up to $80 \mathrm{GHz}$. The red solid lines show the measurement results when there is no absorber. It is used as a reference which proves the necessity of the absorber. By adding a POM absorber, the $3 \mathrm{~dB}$ bandwidth of the fabricated CPW-to-CPS transition increases from $63 \mathrm{GHz}$ to $80 \mathrm{GHz}$.
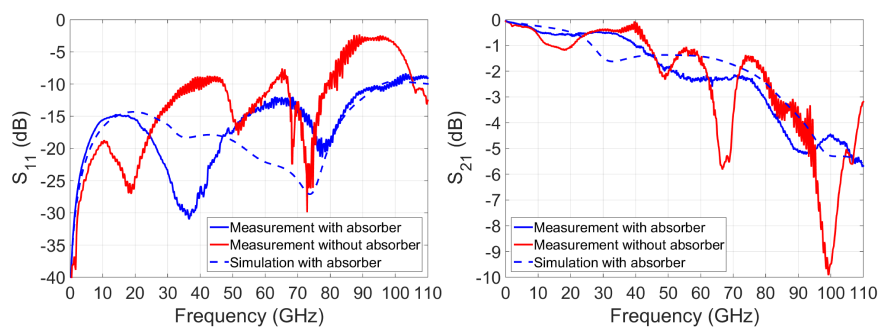

Fig. 11. Measurement results of CPW-to-CPS transition in back-to-back configuration with (blue solid lines) and without absorber layer (red solid lines).

\section{CONCLUSION}

Two types of coplanar transitions based on AIN substrate used for interposer designs of terabit transceivers have been presented. The transitions were designed and simulated in back-to-back configuration with wire bonding bridges and POM absorber. The designs of CCPW, coupled line, CPW, and CPS based on AIN substrate were explained. By adding wire bonding bridges along the transition structures and an POM absorber layer between the AlN substrate and the bottom heat sink layer, parasitic modes were suppressed. For differential signal pair, the CCPW-to-coupled line transition in back-to-back configuration with wire bonding bridges has been demonstrated. The simulated return loss is better than $11 \mathrm{~dB}$ and the insertion loss is less than $2 \mathrm{~dB}$ up to 110 GHz. For single-ended signals, the CPW-to-CPS transition in back-to-back configuration with wire bonding bridges has been designed, fabricated, and measured. A good agreement between simulation and measurement has been achieved. The measured return loss is better than $12 \mathrm{~dB}$ and the insertion loss is less than $3 \mathrm{~dB}$ up to $80 \mathrm{GHz}$. The described CCPWto-coupled line and CPW-to-CPS transitions are sufficient to support a bandwidth of $64 \mathrm{GHz}$ for the terabit transceivers.

\section{ACKNOWLEDGMENT}

The work was supported by the European Commission funded project PANTHER (Contract No. 619411).

\section{REFERENCES}

[1] A. Zapata, M. Duser, J. Spencer, P. Bayvel, I. De Miguel, D. Breuer, N. Hanik, and A. Gladisch. Next-generation 100-gigabit metro ethernet (100 gbme) using multiwavelength optical rings. Lightwave Technology, Journal of, 22(11):2420-2434, Nov 2004.

[2] PANTHER. Fact sheet. http://www.ict-panther.eu/.

[3] H. Avramopoulos, V. Katopodis, P. Groumas, L. Gounaridis, A. Konczykowska, Z. Zhang, G. De Valicourt, R. Dinu, A. Vannucci, T. K. Johansen, A. Pagano, and C. Kouloumentas. Passive and electro-optic polymer photonics and InP electronics integration for multi-flow terabit transceivers at edge SDN switches and data-center gateways. 2014 16th International Conference on Transparent Optical Networks (ICTON), 1-4, July 2014.

[4] Y. Dong, T. K. Johansen, V. Zhurbenko, A. Beretta, A. Vannucci, and G. Locatelli. A 3D hybrid integration methodology for terabit transceivers. Microwave and Optoelectronics Conference (IMOC), 2015 SBMO/IEEE MTT-S International, 1-5, Nov 2015.

[5] D. E. Anagnostou, M. Morton, J. Papapolymerou, and C. G. Christodoulou. A $0-55 \mathrm{GHz}$ coplanar waveguide to coplanar strip transition. Microwave Theory and Techniques, IEEE Transactions on, 56(1):1-6, Jan 2008. 